\title{
THE STARLIKE RADIUS FOR CLASSES OF REGULAR BOUNDED FUNCTIONS ${ }^{1}$
}

\author{
ROBERT W. SANDERS
}

\begin{abstract}
Let $B_{0}(a)$ be the class of all functions $f$ defined on $|z|<1$ such that (i) $f(z)$ is regular, (ii) $|f(z)|<1$, (iii) $f(0)=0$, (iv) $0<\left|f^{\prime}(0)\right|=a<1$. For fixed $R, a \leqslant R<1$, let $B_{0}(a ; R)$ be that subclass having nonzero zeros at $z=z_{k}, k=1,2, \ldots$, such that $\Pi\left|z_{k}\right|=R$. The subclass having no nonzero zeros is designated as $B_{0}(a ; 1)$. A sharp lower bound for $\operatorname{Re}\left[z f^{\prime}(z) / f(z)\right]$ for the class $B_{0}(a ; R), a \leqslant R \leqslant 1$, is obtained, and the radius of starlikeness is found. A covering theorem for the class is also obtained.
\end{abstract}

Introduction. Dieudonné [1, pp. 349-352] obtained the starlike radius for the class $B_{0}(a)$ of functions $f$ which are regular and bounded on the unit disc and which are normalized by (i) $f(0)=0$, (ii) $f^{\prime}(0)=a>0$ and (iii) $|f(z)|$ $\leqslant 1$. Here we will find the starlike radius for a subclass of $B_{0}(a)$. Suppose $f(z) \in B_{0}(a)$ has zeros at $z=z_{k}, k=1,2, \ldots$, where $0<\left|z_{k}\right|<1$ for all $k$. For fixed $R, a \leqslant R<1$, designate as $B_{0}(a ; R)$ the subclass of functions $f$ such that $\prod_{k}\left|z_{k}\right|=R$ and $f\left(z_{k}\right)=0$. For completeness the subclass having no nonzero zeros for $|z|<1$ is designated as $B_{0}(a ; 1)$. The starlike radius will be obtained by finding a sharp lower bound for $\operatorname{Re}\left[z f^{\prime}(z) / f(z)\right]$ on $|z|=r<1$. A covering theorem will also be given.

The following two representation theorems form the basis of the subsequent analysis.

TheOREM. Let $f \in B_{0}(a ; 1)$. Then, for $|z|<1$, there is defined on $[-\pi, \pi] a$ nondecreasing function, $\alpha(t)$, with $\alpha(\pi)-\alpha(-\pi)=1$, such that

$$
f(z)=z \exp \left\{(\log a) \int_{-\pi}^{\pi}\left(1+e^{i t} z\right)\left(1-e^{i t} z\right)^{-1} d \alpha(t)\right\} .
$$

THEOREM. Let $f \in B_{0}(a)$ and let $\left\{z_{k}\right\}$, counted according to their multiplicity, be the nonzero values where $f(z)=0$. Then $f$ is uniquely expressible in the form

$$
f(z)=h(z) \prod\left(\frac{\bar{z}_{k}}{\left|z_{k}\right|} \cdot \frac{z_{k}-z}{1-\bar{z}_{k} z}\right), \quad|z|<1,
$$

where $h(z) \in B_{0}(a / R ; 1)$ and $R=I I\left|z_{k}\right|$.

Received by the editors June 14, 1974 and, in revised form, March 19, 1975.

AMS (MOS) subject classifications (1970). Primary 30A32; Secondary 52-01, 52A40.

Key words and phrases. Starlike radius, bounded functions.

${ }^{1}$ This paper is based on the author's dissertation submitted to Arizona State University for the Ph.D degree. The author wishes to express his gratitude to Professor W. T. Scott for his valuable suggestions and guidance. 
The first theorem is proved by applying Herglotz's Theorem (cf. [6, p. 275]) to

$$
F(z)= \begin{cases}\log [f(z) / z], & z \neq 0 \\ \log a, & z=0\end{cases}
$$

The second theorem is an adaptation of the Blaschke representation theorem(cf. [4, pp. 63-67]).

Preliminary relations. The following relations, which are used in the derivation, are interesting in themselves.

Lemma. Let $\left\{t_{k}\right\}, 0<t_{k}<1$, be given such that $\Pi t_{k}=T>0$. Then

$$
0 \leqslant 1 / T-T-\sum\left(1 / t_{k}-t_{k}\right) .
$$

Equality occurs only if $\left\{t_{k}\right\}=\{T\}$. This result is the best possible in the sense that for any $T, 0<T<1$, there exists an admissible sequence $\left\{t_{k}\right\}$, either finite (with any specified nonzero number of elements) or infinite, such that the right side of (3) is arbitrarily small.

The inequality can be proved by mathematical induction for the finite case and by a limit process for the infinite case. That the result is "best possible" can be demonstrated by noting that, for any sequence $\left\{t_{k}\right\}$ having $t_{1}=T+$ $\theta(1-T), 0<\theta<1$, and $\Pi t_{k}=T$,

$$
\frac{1}{T}-T-\sum\left(\frac{1}{t_{k}}-t_{k}\right)<\frac{1}{T}-T-\left(\frac{1}{t_{1}}-t_{1}\right)<\frac{\theta}{T^{2}} .
$$

Inequality (3) is equivalent to the following

THEOREM. Let $\left\{r_{k}\right\}, 0<r_{k}<1$, with $\Pi r_{k}=R>0$ be given. For each $r$, $0 \leqslant r<R$,

$$
0 \leqslant \frac{1-R^{2}}{(R-r)(1-R r)}-\sum \frac{1-r_{k}^{2}}{\left(r_{k}-r\right)\left(1-r_{k} r\right)} .
$$

Equality occurs only if $\left\{r_{k}\right\}=\{R\}$. This result is the best possible in the sense of the previous Lemma.

We first expand the right side of (5) in a Taylor series. Thus, we want to show

$$
0 \leqslant \sum_{j} r_{j}\left[\frac{1}{R^{j+1}}-R^{j+1}-\sum_{k}\left(\frac{1}{r_{k}^{j+1}}-r_{k}^{j+1}\right)\right] .
$$

We associate $r_{k}^{j+1}$ and $R^{j+1}$ with $t_{k}$ and $T$ of (3) to complete the proof. A result similar to (4) shows (5) is best possible.

(5) can be integrated with respect to $r$ to give

$$
\Pi\left(r_{k}-r\right)\left(1-r_{k} r\right)^{-1} \geqslant(R-r)(1-r R)^{-1}, \quad 0 \leqslant r<R .
$$

The starlike radius. The starlike radius $\rho$ for a class of functions is the least upper bound of radii $r$ of discs $|z| \leqslant r$ that are mapped by an arbitrary function $f(z)$ of the class onto a domain having the property that $\arg f(z)$ 
always increases as the point $f(z)$ moves around the boundary in the positive direction. Since $\partial[\arg f(z)] / \partial \theta$ equals $\operatorname{Re}\left[z f^{\prime}(z) / f(z)\right]$, where $z=r \exp (i \theta)$, we must find the values $r$ for which $\operatorname{Re}\left[z f^{\prime}(z) / f(z)\right] \geqslant 0$ on $|z| \leqslant r$ (cf. [2, p. 167]).

It can be shown, by means of (1), that if $f \in B_{0}(a ; 1)$ then, for $|z| \leqslant r<1$,

$$
\operatorname{Re}\left[z f^{\prime}(z) / f(z)\right] \geqslant 1+2 r(1-r)^{-2}(\log a) .
$$

That this result is sharp can be demonstrated by the function

$$
f(z)=z \exp \left\{(\log a)(1+z)(1-z)^{-1}\right\} .
$$

This knowledge of $B_{0}(a ; 1)$ can be used with the Blaschke product to give the main result of this section.

THEOREM. Let $f \in B_{0}(a ; R)$. Then, for $|z| \leqslant r<R \leqslant 1$,

$$
\operatorname{Re}\left[z f^{\prime}(z) / f(z)\right] \geqslant 1+\frac{2 r}{(1-r)^{2}} \log \frac{a}{R}-\frac{r\left(1-R^{2}\right)}{(R-r)(1-R r)} .
$$

This result is sharp.

For a given set $\left\{z_{k}\right\}, 0<\left|z_{k}\right|=r_{k}<1$, such that $\Pi r_{k}=R>0$, let

$$
g(z)=\prod\left(\frac{\bar{z}_{k}}{\left|z_{k}\right|} \cdot \frac{z_{k}-z}{1-\bar{z}_{k} z}\right) \text {. }
$$

Then, for $r<g \cdot 1 \cdot b \cdot r_{k}$, on $|z|=r$, we can show

$$
\operatorname{Re}\left[\frac{z g^{\prime}(z)}{g(z)}\right] \geqslant-\sum \frac{r\left(1-r_{k}^{2}\right)}{\left(r_{k}-r\right)\left(1-r_{k} r\right)} \text {. }
$$

Equality occurs if and only if $z=r e^{i \alpha}$ and $z_{k}=r_{k} e^{i \alpha}$ for all $k$ and some real $\alpha$. We can combine (2), (5), (7), and (9) to obtain the result stated.

The radius of starlikeness $\rho$ for the class is the smallest positive zero of the right side of inequality (8). If $R=1$, this value can be obtained explicitly:

$$
\rho\left[B_{0}(a ; 1)\right]=1-\log a-\left[(1-\log a)^{2}-1\right]^{\frac{1}{2}}
$$

[7, pp. 1031-1032].

To consider the class $B_{0}(a)$, note for $a \leqslant R \leqslant 1$,

$$
\begin{aligned}
1+\frac{2 r}{(1-r)^{2}} \log a & \geqslant 1+\frac{2 r}{(1-r)^{2}} \log \frac{a}{R}-\frac{r\left(1-R^{2}\right)}{(R-r)(1-R r)} \\
& \geqslant 1-\frac{r\left(1-a^{2}\right)}{(a-r)(1-a r)}
\end{aligned}
$$

TheOrem. Let $f \in B_{0}(a)$. Then, for $|z| \leqslant r<a$,

$$
\operatorname{Re}\left[z f^{\prime}(z) / f(z)\right] \geqslant 1-r\left(1-a^{2}\right)[(a-r)(1-a r)]^{-1} .
$$

This result is sharp.

From this we note that 


$$
\rho\left[B_{0}(a)\right]=\left[1-\left(1-a^{2}\right)^{\frac{1}{2}}\right] / a
$$

[1, pp. 349-352].

The following theorem shows the monotonicity of the starlike radius.

ThEOREM. Let $a, R_{1}, R_{2}, 0<a \leqslant R_{1}<R_{2} \leqslant 1$, be given. Then

$$
\rho\left[B_{0}(a)\right] \leqslant \rho\left[B_{0}\left(a ; R_{1}\right)\right]<\rho\left[B_{0}\left(a ; R_{2}\right)\right] .
$$

Covering THEOREM. If $D$ is a point set in the complex plane, $f(z)$ is said to cover $D$ univalently in $K$ if $f$ is univalent in a subregion $K_{1}$ of $K$ and $f\left(K_{1}\right)$ covers $D[5$, p. 374].

If $f \in B_{0}(a ; 1)$, then, for $|z|=r<1$, as a direct consequence of (1), we obtain

$$
r \exp \left\{(\log a) \frac{1+r}{1-r}\right\} \leqslant|f(z)| \leqslant r \exp \left\{(\log a) \frac{1-r}{1+r}\right\} .
$$

When this inequality is used in conjunction with the Blaschke product representation and inequality (6), the following results:

THEOREM. If $f \in B_{0}(a ; R), a \leqslant R \leqslant 1$, then the map of $|z|<1$ by $w=f(z)$ covers univalently the disc

$$
|w|<\rho(R-\rho)(1-R \rho)^{-1} \exp \left\{(\log a / R)(1+\rho)(1-\rho)^{-1}\right\},
$$

where $\rho=\rho\left[B_{0}(a ; R)\right]$. This result is sharp in that no larger disc, centered at the origin, is univalently covered by all $f$ of the class.

The function demonstrating sharpness is

$$
f(z)=z \frac{R-z}{1-R z} \exp \left\{(\log a) \frac{1+z}{1-z}\right\} .
$$

These results show that the radius of starlikeness for the class is also the radius of univalence.

\section{BIBLIOGRAPHY}

1. J. Dieudonné (1931), Recherches sur quelques problèmes rélatifs aux polynômes et aux fonctions bornées, Ann. Sci. Ecole Norm. Sup. (3) 48, 247-358.

2. G. M. Goluzin (1969), Geometric theory of functions of a complex variable, GITTL, Moscow, 1952; English transl., Transl. Math. Monographs, vol. 26, Amer. Math. Soc., Providence, R.I., p. 167. MR 15, 112; 40 \#308.

3. E. Hille (1962), Analytic function theory. Vol. II, Introductions to Higher Math., Ginn, Boston, Mass., 1962, pp. 240, 323. MR34 \# 1490.

4. K. Hoffman (1962), Banach spaces of analytic functions, Prentice-Hall Ser. in Modern Analysis, Prentice-Hall, Englewood Cliffs, N.J. MR24 \# A2844.

5. M. Robertson (1936), On the theory of univalent functions, Ann. of Math. 37, 374408.

6. H. S. Wall (1948), Analytic theory of continued fractions, Van Nostrand, Princeton, N.J., pp. 275-276. MR10, 32.

7. M. Ziegler (1971), Solutions of advanced problems. Best radii of univalency, Amer. Math. Monthly 78, 1031-1032.

Department of Mathematics, Oral Roberts University, Tulsa, Oklahoma 74136 